\title{
The spin content of the proton in full $\mathrm{QCD}^{* \dagger}$
}

\author{
B. Allés ${ }^{\mathrm{a}}$, G. Boyd ${ }^{\mathrm{b}}$ M. D’Elia ${ }^{\mathrm{c}}$ and A. Di Giacomo ${ }^{\mathrm{c}}$ \\ aDipartimento di Fisica, Sezione Teorica, Università degli Studi di Milano and INFN, Via Celoria 16, \\ 20133 Milano, Italy. \\ ${ }^{\mathrm{b}}$ Center for Computational Physics, University of Tsukuba, Tsukuba, Ibaraki 305, Japan \\ ${ }^{\mathrm{c}}$ Dipartimento di Fisica, Università di Pisa and INFN, Piazza Torricelli 2, 56126 Pisa, Italy
}

We present preliminary results on the proton spin structure function in full QCD. The measurement has been done using 4 flavours of staggered fermions and an improved definition of the lattice topological charge density.

\section{INTRODUCTION}

The so-called proton spin crisis is still calling for a theoretical explanation in the context of QCD. The problem may be stated as follows. Let $j_{\mu}^{5}$ be the singlet axial current

$j_{\mu}^{5}=\sum_{i=1}^{N_{f}} \bar{\psi}_{i} \gamma_{\mu} \gamma_{5} \psi_{i}$.

Its on-shell nucleon matrix element may be written as

$$
\begin{aligned}
& \left\langle\boldsymbol{p}, s\left|j_{\mu}^{5}\right| \boldsymbol{p}^{\prime}, s^{\prime}\right\rangle= \\
& \bar{u}(\boldsymbol{p}, s)\left[G_{1}\left(k^{2}\right) \gamma_{\mu} \gamma_{5}-G_{2}\left(k^{2}\right) k_{\mu} \gamma_{5}\right] u\left(\boldsymbol{p}^{\prime}, s^{\prime}\right),
\end{aligned}
$$

where $k=p^{\prime}-p$. In the naive parton model $\Delta \Sigma=G_{1}(0)$ can be interpreted as the fraction of the nucleon spin carried by the quarks, and so is expected to be close to 1 . On the contrary, experimental determinations [1] 3] lead to a value $\Delta \Sigma \sim 0.2$, which is also in contrast with the OZI expectation $\Delta \Sigma \sim 0.7$ [4]. It is the small experimental value of $\Delta \Sigma$ which is referred to as the proton spin crisis.

A numerical simulation on the lattice is the ideal nonperturbative tool to obtain an estimate of $\Delta \Sigma$ from first principles. A direct computation of the matrix element of eq. (2) involves the evaluation of a disconnected diagram, characterized

\footnotetext{
*Preprint numbers IFUP-TH 41/97 and IFUM-581/FT.

${ }^{\dagger}$ Partially supported by EC Contract CHEX-CT92-0051 and by MURST.

${ }^{\ddagger}$ Speaker at the conference.
}

by a very noisy signal [5] 7 . We shall adopt a different method 8 10], which makes use of the axial anomaly

$\partial^{\mu} j_{\mu}^{5}(x)=-2 N_{f} Q(x)$

$N_{f}$ is the number of flavours and

$Q(x)=\frac{g^{2}}{64 \pi^{2}} \epsilon^{\mu \nu \rho \sigma} F_{\mu \nu}^{a}(x) F_{\rho \sigma}^{a}(x)$

is the topological charge density. The on-shell nucleon matrix element of $Q(x)$ can be written, using Eq. (3), as

$$
\begin{aligned}
& \left\langle\boldsymbol{p}, s|Q(x)| \boldsymbol{p}^{\prime}, s^{\prime}\right\rangle= \\
& \quad=\frac{m_{N}}{N_{f}} A\left(k^{2}\right) \bar{u}(\boldsymbol{p}, s) i \gamma_{5} u\left(\boldsymbol{p}^{\prime}, s^{\prime}\right),
\end{aligned}
$$

where $m_{N}$ is the nucleon mass and $A\left(k^{2}\right)=$ $G_{1}\left(k^{2}\right)+G_{2}\left(k^{2}\right) k^{2} / m_{N}$, whence $A(0)=\Delta \Sigma$. The last relation does not hold in the quenched approximation, since $G_{2}\left(k^{2}\right)$ develops a pole at $k^{2}=0$ in that case [9].

Therefore, $\Delta \Sigma$ can be calculated on the lattice by evaluating the nucleon matrix element of the topological charge density $Q(x)$ in full QCD. However, a necessary condition to do this is to have the correct sampling of topological modes. Here we will give some preliminary results obtained using the standard HMC algorithm with four flavours of dynamical staggered flavours and will show that the feasibility of the determination is affected by the bad sampling of topological modes obtained with the HMC algorithm [11]. 


\section{THE METHOD}

We have simulated four degenerate flavours of staggered fermions at $\beta=5.350$ on a $16^{3} \times 24$ lattice, using the standard HMC algorithm described in Ref. [12 and the Wilson action for the pure gauge sector. A trajectory length $\tau=0.6$ was used, with time step $\delta \tau=0.004$. The value $a \cdot m_{f}=0.01$ was chosen for the fermion mass.

In order to evaluate the matrix element of Eq. (5) we need a lattice regularization $Q_{L}(x)$ of the topological charge density. The renormalization properties of $Q_{L}(x)$ are nontrivial [13]: it mixes with the continuum $Q(x), \partial^{\mu} j_{\mu}^{5}(x)$ as well as with $p(x)=\sum_{i=1}^{N_{f}} m_{i} \bar{\psi}_{i} \gamma_{5} \psi_{i}$. However, it can be shown that the last two operators can be safely neglected in a first approximation [13, so that the following relation holds

$Q_{L}=a^{4} Z_{Q} Q+\mathcal{O}\left(a^{6}\right)$.

We use the improved smeared operators $Q_{L}(x)$ proposed in Ref. [14], and in particular the operator defined at the second smearing level. The value of $Z_{Q}$ can be obtained non-perturbatively using the so-called heating method |15, 16]: the short range fluctuations responsible for the renormalization are thermalized by applying a few updating steps on a discretized classical configuration of charge 1. The thermalization shows up as a plateau in $\left\langle Q_{L}\right\rangle$ plotted against the number of heating steps performed, as is shown in Fig. 1. $Z_{Q}$ is then measured on this plateau. We have obtained $Z_{Q}=0.56 \pm 0.04$ with the values of the parameters used in our simulation.

The next step is to compute the correlation function

$C(t)= \pm \frac{i}{2 N_{f} Z_{Q}|\boldsymbol{p}|}\left\langle\bar{B}(\mathbf{0}, 0) P_{ \pm} Q_{L}(\boldsymbol{p}, \tau) B(\boldsymbol{p}, t)\right\rangle,(7)$

where

$Q_{L}(\boldsymbol{p}, \tau)=\sum_{\boldsymbol{x}} e^{i \boldsymbol{p} \boldsymbol{x}} Q_{L}(\boldsymbol{x}, \tau)$,

and $P_{ \pm}$is the spin projection operator. For $\bar{B}$ we have taken the wall source, after performing a Coulomb gauge fixing on the $t=0$ time slice by means of a steepest-descent algorithm. For $B$ we have taken the usual local baryon operator 10,17] $B=\epsilon_{a b c} \chi^{a} \chi^{b} \chi^{c}$.

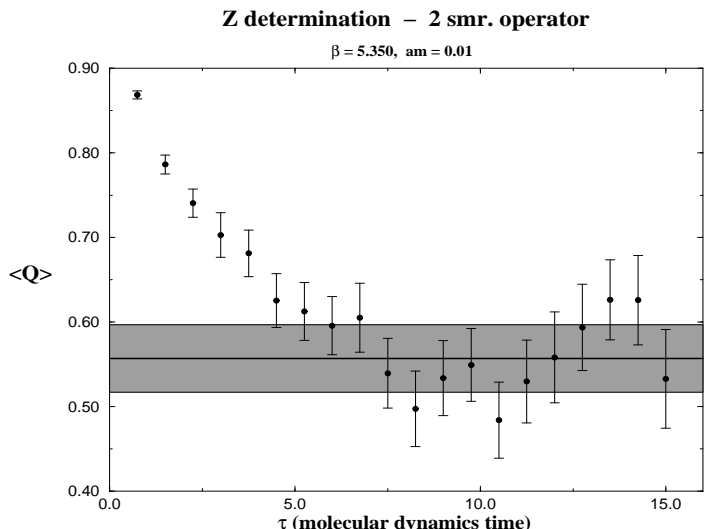

Figure 1. Determination of $Z_{Q}$ for the 2-smeared operator. The number of heating steps performed is measured in terms of molecular dynamics time.

The following behaviour is expected for $C(t)$ [10, 17]

$$
\begin{aligned}
C(t)= & A_{+} \Delta \Sigma e^{-m_{N} \tau-E_{N}(t-\tau)}+ \\
& A_{-} \Delta \Sigma_{\Lambda}(-1)^{t} e^{-m_{\Lambda} \tau-E_{\Lambda}(t-\tau)},
\end{aligned}
$$

where $A_{+(-)}$and $m_{N(\Lambda)}$ can be determined by a fit to the full baryon propagator $\langle\bar{B}(0) B(t)\rangle$, 17]. $\Delta \Sigma_{\Lambda}$ and $m_{\Lambda}$ are the axial baryonic charge and mass of the $\Lambda(1405)$ particle respectively [17].

\section{RESULTS AND CONCLUSIONS}

We have collected a sample of 230 configurations, each separated by $15 \mathrm{HMC}$ trajectories, using 700 hours of a 25 Gflops APE/QUADRICS machine.

In Fig. 2 we show the topological charge distribution in our sample. It is clear that the correct thermalization for the topological charge has not yet been achieved: $\langle Q\rangle \neq 0$ and the distribution is not symmetric under $Q \rightarrow-Q$. The time history of the topological charge is shown in Fig. 3, where the inefficiency of the HMC algorithm to change the topological sector is clearly visible.

Despite this deficiency in our ensemble of configurations, we have attempted an estimate of $\Delta \Sigma$ and $\Delta \Sigma_{\Lambda}$. From a fit to the full baryon propagator we have obtained

$$
\begin{array}{ll}
A_{+}=0.152(12), & m_{N}=0.693(11) \\
A_{-}=-0.192(35), & m_{\Lambda}=0.797(25)
\end{array}
$$




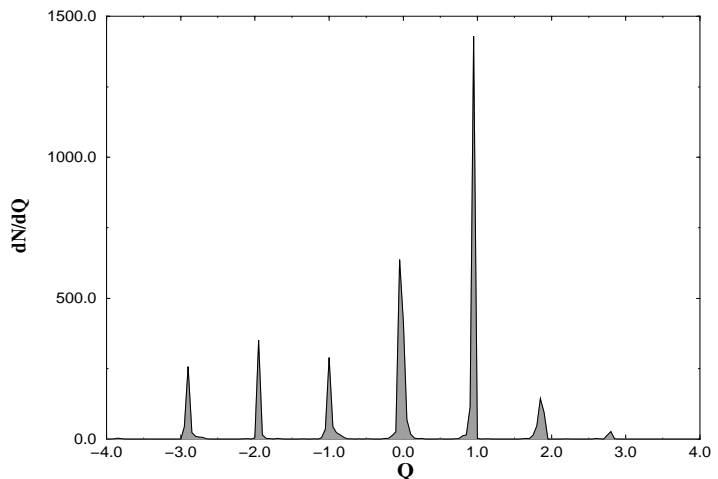

Figure 2. Topological charge distribution after 30 cooling steps.

We have thereafter measured the correlation function of Eq. (7). The zero momentum transfer limit has been approximated by using $|\boldsymbol{p}|=2 \pi / 16$ ( $\sim 600 \mathrm{MeV}$ on our lattice). An average has been performed over the three possible spatial directions for $\boldsymbol{p}$ and over the two possible polarizations.

We have observed a poor signal for $C(t)$ and, by combining data at $\tau=4$ and $\tau=20$, we have obtained

$\Delta \Sigma \simeq 0.04 \pm 0.04, \quad \Delta \Sigma_{\Lambda} \simeq 0.05 \pm 0.05$

The error analysis has been done by a jackknife method. However, the error quoted in Eq. (12) does not take account of the systematic error coming from the bad sampling of topological modes. By looking at the effective number of topological sectors explored during the simulation (Fig. 3), we roughly estimate the effective error to be up to $4-5$ times the one quoted in Eq. (12).

Therefore our data can only be regarded as preliminary and no conclusive results can be produced unless a correct sampling of topological modes is obtained. This goal seems to be hardly feasible for the standard HMC algorithm: the possibility of achieving it by using different algorithms is currently under investigation.

\section{REFERENCES}

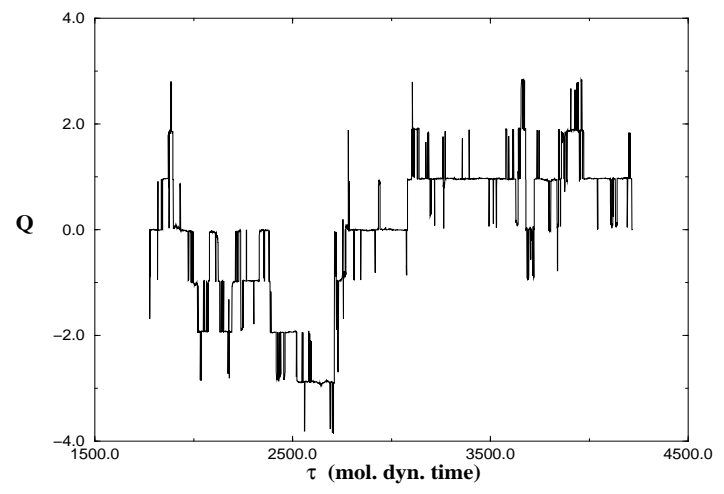

Figure 3. Time history, in units of molecular dynamics time $\tau$, of the topological charge $Q$.

1. EMC, J. Ashman et al., Nucl. Phys. B 328 (1989) 1.

2. SMC, D. Adams et al., Phys. Lett. B 329 (1994) 399.

3. E143, K. Abe et al., Phys, Rev. Lett. 74 (1995) 346.

4. G.M. Shore, G. Veneziano, Nucl. Phys. B 381 (1992) 23.

5. J.E. Mandula, M.C. Ogilvie, Phys, Lett. B 312 (1993) 327.

6. M. Fukugita, Y. Kuramashi, M. Okawa, A. Ukawa, Phys. Rev. Lett. 75 (1995) 2092.

7. S.J. Dong, J.F. Lagaë, K.F. Liu, Phys. Rev. Lett. 75 (1995) 2096.

8. B. Allés et al., Phys. Lett. B 336 (1994) 248.

9. R. Gupta, J.E. Mandula, Phys. Rev. D 50 (1994) 6931.

10. R. Altmeyer et al., Phys. Rev. D 49 (1994) R3087

11. B. Allés, G. Boyd, M. D'Elia, A. Di Giacomo, E. Vicari, Phys. Lett. B 389 (1996) 107.

12. S. Gottlieb et al., Phys. Rev. D 35 (1987) 2531.

13. B. Allés et al., Phys, Lett. B 350 (1995) 70.

14. C. Christou, A. Di Giacomo, H. Panagopoulos, E. Vicari, Phys. Rev. D53 (1996) 2619.

15. A. Di Giacomo, E. Vicari, Phys. Lett. B 275 (1992) 429.

16. B. Allés, M. D'Elia, A. Di Giacomo, Nucl. Phys. B494 (1997) 281.

17. R. Altmeyer et al., Nucl. Phys. B 389 (1993) 
445. 